\title{
Nano-alumina and radiation effect on the mechanical properties of high density polyethylene/hydroxy apatite composite
}

\begin{abstract}
The effect of nano-alumina and radiation on the mechanical properties of high density polyethylene hydroxyl apatite composite was investigated. The study showed that nano alumina as filler in the composite enhanced the strength of the polymer matrix and hence improved the mechanical properties of the composite. The study also showed that the mechanical properties of the composite depended on doses of nano alumina used and radiation dose of gamma ray. The maximum radiation dose used in this experiment $100 \mathrm{KGy}$ is the best dose for the composite that enhances the tensile strength, impact, modulus and flexural strength. The interface behavior and strengthening mechanisms are discussed.
\end{abstract}

Keyword: Hydroxyl apatite; Mechanical properties; Nano alumina; Polyethylene; Radiation 